\title{
Control Automático de Condiciones Ambientales en Domótica usando Redes Neuronales Artificiales
}

\author{
Mauricio R. Henríquez, Patricio A. Palma \\ Universidad Austral de Chile, Escuela de Ingeniería en Computación, Casilla 1327, Puerto Montt- \\ Chile (e-mail: mauriciohenriquez@uach.cl, mail@ppalma.cl)
}

Recibido Nov. 12, 2010; Aceptado Feb. 26, 2011; Versión Final recibida Mar. 15, 2011

\begin{abstract}
Resumen
Se propone el uso del concepto de computación omnipresente para mejorar las relaciones humano-maquina en entornos automatizados como hogares, oficinas y edificaciones. Se registran los patrones de comportamiento del usuario para luego emplearlos en un sistema consciente del contexto, capaz de reaccionar de forma automática y permitiendo la autoconfiguración de un sistema automatizado en un entorno de oficina. Se detalla el mecanismo automatizado por el cual se determina el estado de la iluminación, temperatura, humedad y otros valores de entorno y cómo se actúa sobre ellos. Se considera para ello los hábitos diarios del usuario y las condiciones reales del entorno. Se utilizan Redes de Neuronas Artificiales como clasificador del estado de las luces, calefacción y ventilación. Los resultados muestran que la técnica de redes neuronales es capaz de reconocer satisfactoriamente más de un $90 \%$ de los patrones del usuario.
\end{abstract}

Palabras clave: control automático, redes neuronales, reconocimiento de patrones, computación omnipresente.

\section{Automatic Control of Environmental Conditions in Domotics using Artificial Neural Networks}

\begin{abstract}
It is proposed to use the concept of ubiquitous computing for improving human-machine relations in automated homes, offices and building environments. The patterns behavior of the user is registered to use them in a context-aware, able to automatically react and allowing autoconfiguration of an automated system in an office environment.

The automated mechanism which determines the state of lighting, temperature, humidity and other environment values, and how the system acts on them, are detailed. The daily habits of the user and the actual conditions of the environment are considered. Artificial Neural Networks are used to classify the state of light, heating and ventilation. The results show that the neural network technique is fully capable of recognizing more than $90 \%$ of the user patterns.
\end{abstract}

Keywords: automatic control, neural systems, pattern recognition, ubiquitous computing 


\section{INTRODUCCION}

El concepto de computación omnipresente puede aplicarse a sistemas de automatización de residencias y lugares de trabajo, dotando al sistema de la habilidad tanto para reaccionar ante las acciones del usuario y aprender de sus hábitos, como para controlar aspectos ligados al ahorro energético, confort y seguridad del entorno. Sistemas de computación ubicua (omnipresente), como los presentados por Mark Weiser, tienen como objetivo desarrollar aplicaciones que reaccionan ante situaciones, facilitando tareas cotidianas y permitiendo que el uso de la tecnología se produzca sin esfuerzo, mejorando las relaciones humano-maquina (Weiser, 2007). La importancia de aplicar estos conceptos radica en que las personas se ven cada día rodeadas de más tecnologías, las cuales en muchos casos no son lo suficientemente simples. Es así que los sistemas de automatización están cada vez más disponibles como una característica estándar en hogares, oficinas y edificios en general. Por tanto, el desarrollo de ambientes automatizados dotados de real inteligencia, capaz de adaptarse por un lado al uso que se les da y por otro mantener condiciones propicias que apoyen la seguridad y el ahorro energético, son características esenciales en los futuros sistemas de éste tipo.

Desde hace algunos años, diferentes entornos inteligentes dedicados se han estado desarrollando en empresas y Universidades en todo el mundo, por ejemplo, la investigación multidisciplinaria llevada a cabo por la Universidad de Texas MavHome (Cook,2003), enfocada en la creación de un ambiente de hogar inteligente; el proyecto AwareHome (Kidd,1999) implementa un laboratorio en vivo para el estudio de las actividades diarias y computación ubicua; iniciativas conjuntas como PlaceLab (Larson,2005), llevada a cabo por la empresa TIAX y el Instituto de Tecnología de Massachusetts (MIT) desarrollan un laboratorio viviente para el estudio de la conducta humana, interacción y rutinas; la empresa Phillips fundó HomeLab (Philips,2008), como un escenario de pruebas en tecnologías de interacción en ambientes residenciales. El reconocimiento de las Actividades de la Vida Diaria (AVD), se enfoca en investigaciones ligadas a lograr alta precisión del contexto y forma en que se realizan las actividades, para luego utilizar esta información en aplicaciones de alto nivel. El trabajo desarrollado por Carlos Machado y José A. Mendes (Machado, 2007), se enfoca en el desarrollo de clasificadores RNA para el control de iluminación en entornos de Domótica. Estudios más recientes como el llevado a cabo por Natalie Kcomt Ché (Kcomt, 2010) y el desarrollado por la Universidad Politécnica de la Marche, Italia (Morganti, 2009), se enfocan en la predicción de las acciones de los usuarios en entornos automatizados.

Diversas técnicas de minería de datos se han empleado en los últimos años para procesar la información capturada desde una serie de sensores distribuidos en el entorno con el propósito de determinar patrones en la actividad humana, Tapia et al. (Tapia, 2004) utiliza un clasificador Bayesiano para reconocer AVD como vestirse, preparar el almuerzo o bañarse, analizando los datos capturados mediante una serie de sensores simples de cambio de estado; en (Kautz,2003), se utiliza en parte un modelo oculto de Markov para hacer un seguimiento de las actividades diarias de las personas en un conjunto de residencias asistidas; se emplea un agente temporal basado en redes neuronales (Rivera,2006) para reconocer comportamientos AVD como escuchando música, trabajando con el computador o durmiendo; se utiliza una interfaz de usuario (IU) para etiquetar las actividades de los usuarios; Luithr et al. (Luihr,2007), emplea minería de datos mediante reglas asociativas de intertransacciones (RAI), para la detección de cambios 0 nuevos comportamientos de las personas que viven en una casa inteligente; en (Zheng,2008) se utiliza una red neuronal auto-adaptativa llamada Mapas de Crecimiento Auto-Organizados (MCAO), para un análisis por agrupamiento de las actividades humanas diarias.

Los proyectos antes mencionados, estudian el comportamiento de individuos en entornos residenciales tipo casa o departamento, este estudio se enfoca en los hábitos de los usuarios en ambientes de oficina preocupándose principalmente del confort y ahorro energético. Hay varias razones para este cambio de enfoque, pero el principal impulsor es la posibilidad de comercialización. Las tecnologías de domótica requieren de un considerable esfuerzo de fabricación y distribución masiva (uno de los principales problemas de éste tipo de tecnologías), y están sujetos a las tendencias de los consumidores, lo cual es un riesgo considerable para una 
primera versión de un potencial producto. Un enfoque más corporativo, como un entorno de oficina, presenta un mercado objetivo potencialmente más atractivo para cualquier posible solución, donde el potencial comprador sería una unidad económica que cubre una amplio número de oficinas de una sola vez, y al mismo tiempo ofrece a cualquier cliente potencial un valor añadido y ventaja de marketing a través de espacios de trabajo totalmente automatizado y con un uso eficiente de la energía.

En este trabajo, se presenta un método completamente autónomo usando RNA para determinar los patrones de los usuarios respecto a la utilización de las luces, calefacción, estado de la puerta y ventana (abierta/cerrada), con el fin de predecir su estado y actuar sobre ellas de forma automática. El presente método no necesita de ninguna intervención directa del usuario en la Interfaz de Usuario (IU). Sólo usa los patrones adquiridos en el uso normal de los usuarios de las luces, calentador, ventilador, etc, y por tanto, no requiere la tarea de etiquetado. Se dotó a la oficina instrumentada de una serie de sensores y actuadores que permitieron una captura real de los datos del entorno y su posterior control.

\section{ADQUISICION DE DATOS}

Para un sistema de reconocimiento de patrones, es fundamental en primer lugar la captura de datos, es así como lo explica Polikar (Polikar, 2006). A diferencia de los estudios mencionados anteriormente, los cuales se enfocan en detectar los patrones de uso de dispositivos en entornos residenciales, en éste proyecto, los datos fueron recogidos desde un entorno de oficina instrumentada donde un empleado desempeña sus labores. El sistema controla el uso completo de luces, temperatura, humedad y el estado de la puerta y ventana de la oficina, las 24 horas del día, desde agosto de 2010.

Es importante que la adquisición de los datos relacionados con las acciones del usuario se realice tanto de manera implícita como de forma centralizada, es decir por un lado de manera que el usuario no perciba el proceso de captura de los datos, interactuando de forma normal con los dispositivos de la oficina (luces, calentador, ventilador, puerta y ventana) (Schmit, 1999), y a su vez permitiendo el control de forma centralizada de estos para brindar un mayor confort al momento de utilizarlos. Estos aspectos fueron considerados en el hardware que se muestra en la figura 1 , los cuales controlan los instrumentos de la oficina.

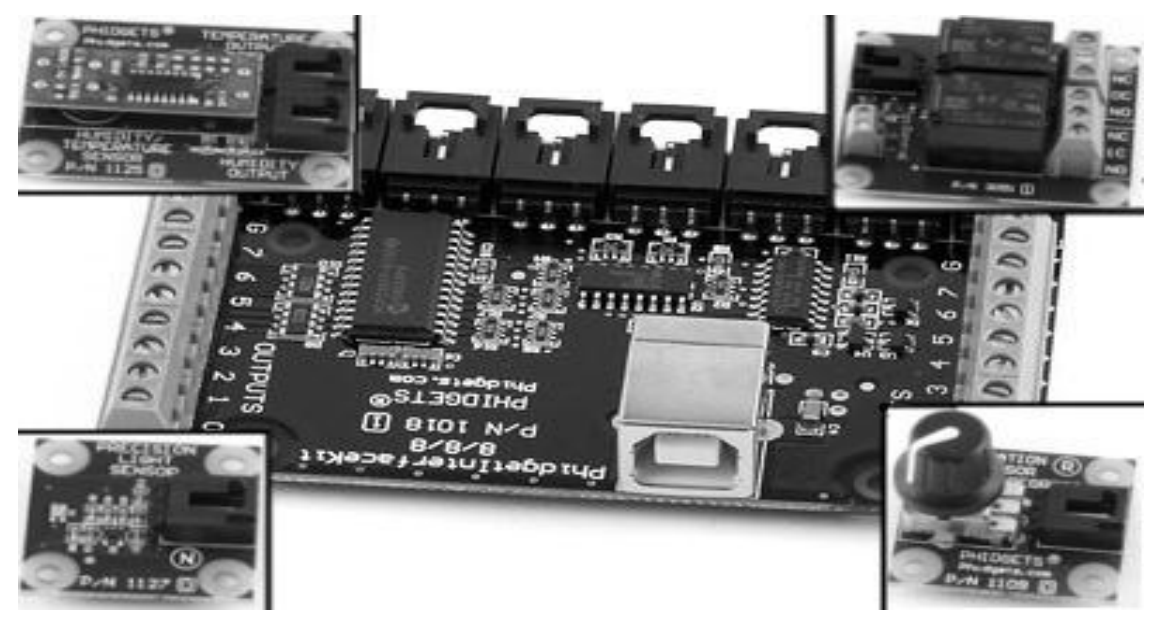

Fig. 1: Interfaz Phidgets 8/8/8, Sensores y Relés.

Se utilizó la "Interfaz8/8/8" de la empresa Phidgets (Phidgets, 2010), la cual permite la conexión de 8 sensores análogos, 8 salidas digitales y 8 entradas digitales. Se conectaron sensores de luz, temperatura, humedad, ultrasonido y de rotación para la puerta y ventana a las entradas análogas. Las salidas digitales se conectaron a relés para controlar el encendido o apagado de las lámparas, calefacción, ventilación y la posición de la puerta y ventana en la oficina instrumentada. Y las entradas digitales se utilizaron como interruptores normales de estos aparatos, para simular su accionar de forma regular e implícita. 
Por el lado del software, se construyó una escena tridimensional que se asemeja a la oficina real, tal como se muestra en la figura 2. Esto mediante el motor para escenarios 3D Icarus (Icarus, 2010) y el marco de trabajo (Framework) para automatización y robótica monoBOTICS (monoBOTICS, 2010), los cuales no solo permitieron la creación de la escena de realidad virtual (Hollands,1996 ), sino que también vincular los objetos 3D con los dispositivos reales de la oficina, permitiendo ver en tiempo real las acciones de los usuarios en la oficina real en el entorno virtual y a su vez, controlar los dispositivos reales desde el entorno virtual (Ej.: encender la lámpara real enciende la lámpara virtual y apagar la lámpara virtual apaga la lámpara real). El software permitió de esta forma registrar las acciones de los usuarios sobre los dispositivos monitoreados (lámparas, calentador, ventilador, puerta y ventana), además de las condiciones ambientales reales del entorno: lumen, grados Celsius, humedad relativa, grado de apertura/cierre de puerta/ventana, entre otros. Las características del hardware y software le permiten a los componentes instalados en la oficina instrumentada, ser incluidos en un sistema de automatización omnipresente como se describe en (Machado, 2007).

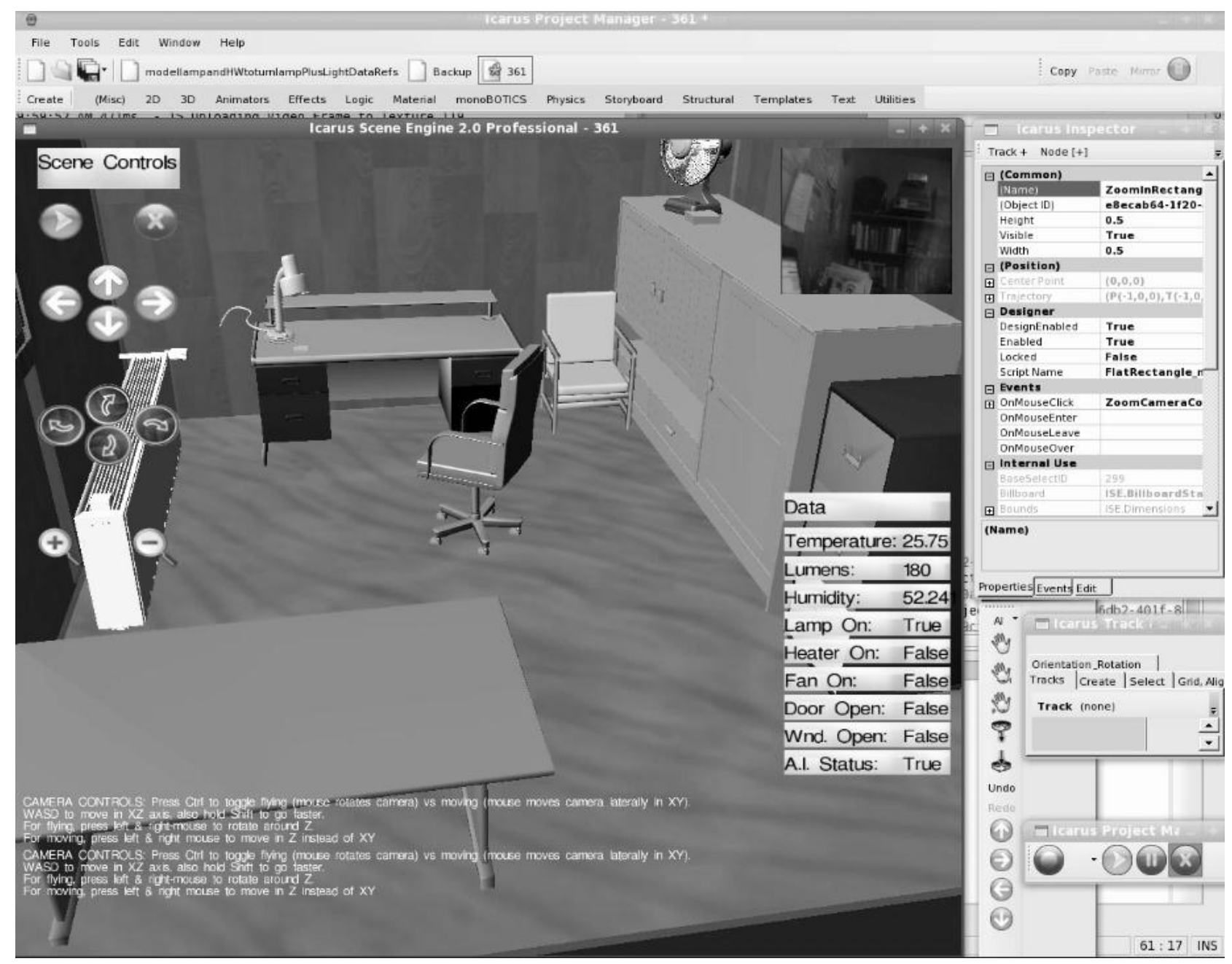

Fig. 2: Interfaz Simulación Icarus + monoBOTICS.

Los eventos son capturados por la interfaz Phidgets y luego son transmitidos a un computador central donde se registra el tiempo de la muestra (Machado, 2007). Por último, los registros se almacenan en una base de datos. La tabla 1 muestra un ejemplo de los registros que se capturan sobre las acciones de los usuarios y las condiciones del entorno, se capturan cada 30 segundos 0 menos dependiendo de la resolución deseada. 
Tabla 1: Ejemplos de eventos registrados.

\begin{tabular}{|l|c|c|c|c|c|c|c|c|c|c|c|}
\hline Tiempo & Lumen & $\mathrm{T}^{\circ} \mathrm{C}$ & Hum. & Personas & $\begin{array}{r}\text { Giro } \\
\text { Puerta }\end{array}$ & $\begin{array}{r}\text { Giro. } \\
\text { Ventana }\end{array}$ & Lámpara & Ventilador & Calentador & Puerta & Ventana \\
\hline 8:55am & 43 & 19 & 44.9 & 0 & 122.7 & 42.9 & False & False & False & False & False \\
\hline 9:05am & 109 & 19 & 46.9 & 1 & 250.8 & 42.9 & True & True & False & True & False \\
\hline 6:30pm & 86 & 24 & 47.4 & 1 & 250.8 & 42.6 & True & True & True & True & False \\
\hline 6:40pm & 9 & 21.5 & 49.3 & 0 & 101.1 & 24.6 & False & False & False & False & False \\
\hline
\end{tabular}

Cada registro muestra el estado actual de los dispositivos monitoreados (lámpara, calentador, ventilador, puerta y ventana), así como la presencia o no de personas en el lugar (lo cual se deduce a partir de información de sensores de ultrasonido y análisis de imágenes en tiempo real). También se registran las condiciones de luz, humedad, temperatura y rotación de puerta/ventana de la oficina instrumentada. El primer evento se registró a las 8:55am y muestra un bajo estado de lumen en el ambiente y la totalidad de los dispositivos apagados, puesto que en ese momento no hay ninguna persona en la oficina. El segundo evento a las 9:05am (hora de llegada del usuario de la oficina), muestra la puerta como abierta (True) y un incremento en los lúmenes del entorno asociado al encendido de la lámpara. De forma similar, los demás eventos muestran el estado de los dispositivos y condiciones del entorno en diferentes horarios. La información contenida en los registros, puede ser utilizada para obtener información mediante técnicas de minería de datos, cálculo de datos estadísticos o como conjuntos de entrenamiento para aprendizaje de máquinas.

En la figura 3 se presentan los gráficos de la media en cuanto a luminosidad (lumen) y ángulo de apertura/cierre de la puerta de la oficina instrumentada, con una resolución de dos muestreos por minuto en un periodo de 60 días, diferenciando los datos de los días de la semana de los del fin de semana. En cuanto a los lúmenes, podemos apreciar que el rango mayor a 100 y menor a 250, se encuentra en el horario de uso normal de la oficina entre las $9 \mathrm{Hrs}$ y las $18 \mathrm{Hrs}$ para los días de la semana de lunes a viernes y entre 100 y 200 lumen el fin de semana en el horario alrededor de las $16 \mathrm{Hrs}$ (horario de aseo de la oficina). Esto muestra que la cantidad de luminosidad adecuada para el uso regular de la oficina cuando hay personas dentro está en el rango de los 100 a 250 lumen en general. El ángulo de apertura de la puerta aumenta en el horario en donde el empleado o el personal de aseo hace uso de la oficina, es decir en el horario entre las $9 \mathrm{Hr}$ a $18 \mathrm{Hrs}$ para los días de la semana y entre las $16 \mathrm{Hr}$ y $17 \mathrm{Hrs}$ para el fin de semana, puesto que para el caso de la oficina instrumentada, el empleado acostumbra a mantener su puerta abierta en los momentos en los que él se encuentra. Se realizó el mismo análisis para los datos capturados respecto a la temperatura de acuerdo a las condiciones de uso luego de un periodo de muestreo, para la cual se determinó un rango óptimo de entre $18^{\circ}$ y $22^{\circ}$ grados Celsius y las condiciones de humedad relacionadas con el estado abierto o cerrado de la ventana en la oficina instrumentada.

Los histogramas que se muestran en la figura 4 señalan los intervalos de lúmenes en los cuales el usuario enciende o apaga la luminaria de la oficina y también los intervalos de horario en los cuales el empleado mantiene su puerta abierta o cerrada. 

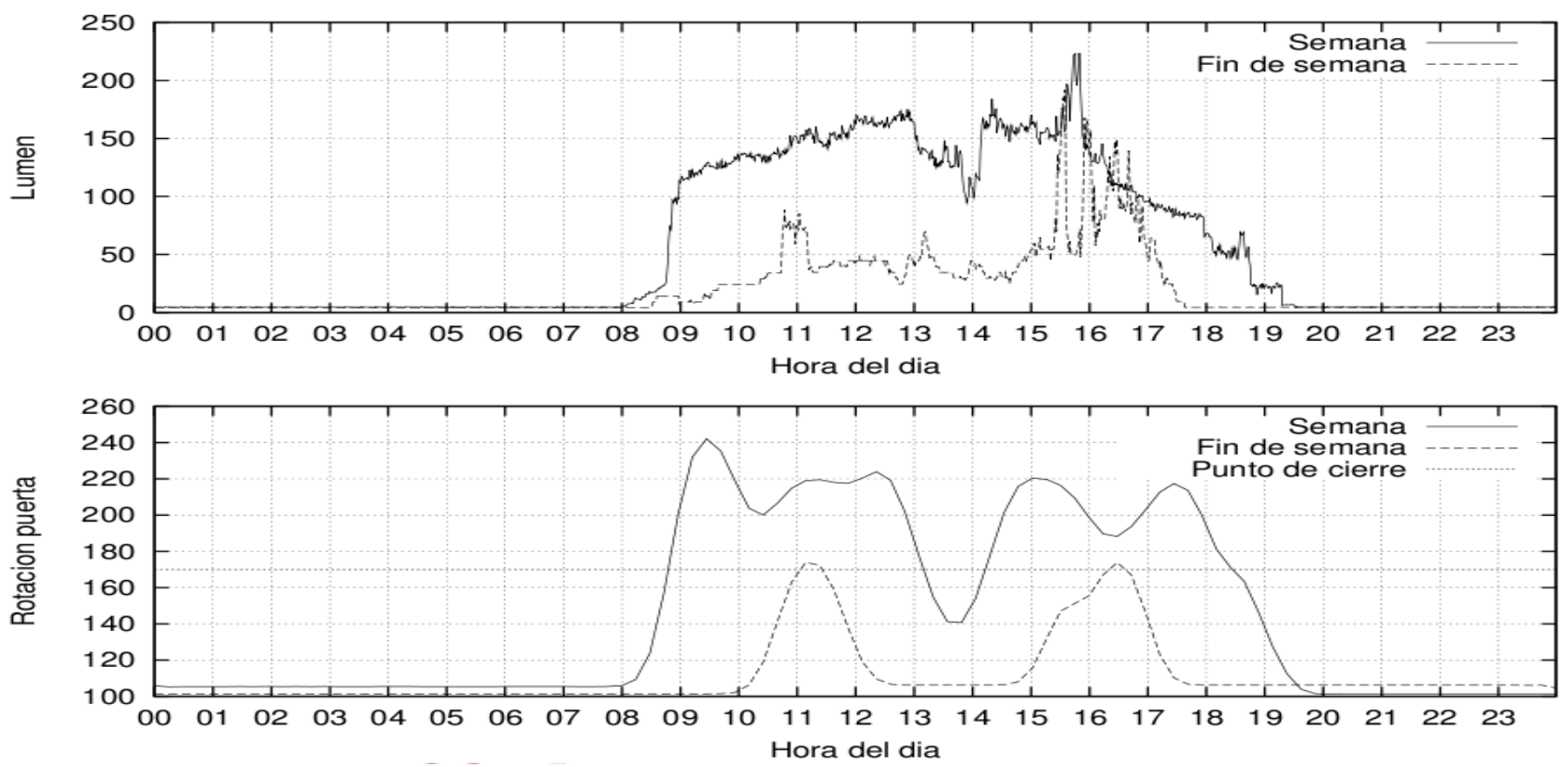

Fig. 3: Promedio luminosidad y ángulo de puerta con resolución cada 1/2 minuto (periodo de 60 días)

La importancia de estos gráficos radica en que nos permite determinar los rangos de lúmenes u horarios en los cuales es más probable que el usuario haga uso ya sea de las lámparas o abra/cierre la puerta respectivamente. Es posible ver que casi el $90 \%$ de las veces que se encendió la luminaria de la oficina, se realizó cuando la luminosidad ambiental natural estaba entre 21 y 40 lúmenes y casi el $100 \%$ de las veces que se apagó la luminaria, los lúmenes estaban en el rango de los 281 a 300 . Los lúmenes que las lámparas de la oficina aportan o disminuyen es de alrededor de 100, lo que nos permite confirmar que el rango de iluminación que al usuario le parece adecuada para realizar su trabajo es de 100 a 250 lúmenes. El análisis del histograma de uso de la puerta, nos permite determinar en qué rangos horarios ésta permanece abierta o cerrada. El gráfico muestra que los mayores porcentajes de apertura se ubican en los horarios entre las 9:00am y 01:00pm y luego entre las 02:30pm y 06:30pm y permanece cerrada un alto porcentaje de los demás horarios.

Se realizaron análisis análogos para el estudio de los rangos adecuados de temperatura y humedad, determinando la frecuencia de uso de la calefacción según rangos de temperatura y apertura/cierre de la ventana según rangos de humedad. Esta información, nos permite determinar los rangos adecuados en los cuales el sistema debe fijar su atención para mantener a la oficina en condiciones de luz, temperatura y estado de puerta y ventana de acuerdo a los gustos/patrones del usuario. Dentro de este análisis, se deben considerar las diferencias entre el uso normal de la oficina instrumentada durante los días de la semana de los del fin de semana. Para mejorar la estabilidad del sistema resultante, cualquier cambio de estado que de menos de 30 segundos fue descartado. Esto se utilizo para permitir los cambios de estado temporal por el usuario, y también para superar algunos valores erradamente informados por el Hardware. Se recomienda que este valor sea equilibrado según el ambiente en particular. 

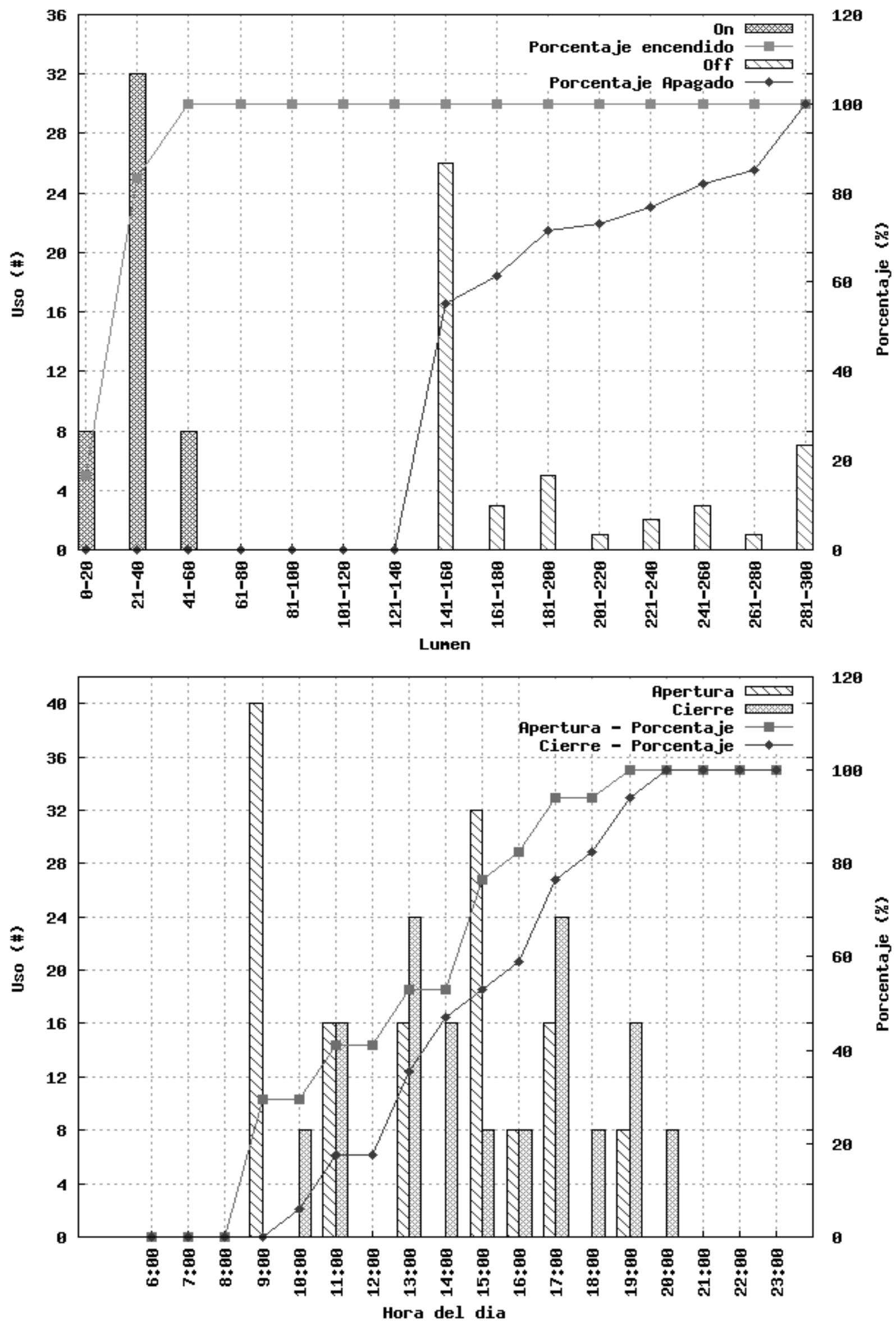

Fig. 4: Histograma uso de iluminación en rangos de lúmenes y apertura de puerta en rangos horarios (60 días) 


\section{DATOS CAPTURADOS}

Los datos capturados indican que el uso de las luces en cierto rangos de iluminación y lo mismo para la calefacción en ciertos rangos de temperatura, están de alguna manera relacionados y siguen un patrón. Esto significa que es posible predecir el estado adecuado de una lámpara mirando los rangos de iluminación presentes y que es posible determinar el estado encendido o apagado de la calefacción mirando los rangos de temperatura registrados, lo mismo para el estado de la puerta y ventana. Para esto, es también necesario tener en cuenta la hora y día de la semana, así como la presencia o no de personas en la oficina con miras al ahorro energético, puesto que si no hay personas en su interior, no es necesario mantener la iluminación ni la temperatura en ciertos rangos y la puerta y ventana deben permanecer cerradas. La hora y día de la semana ayudará al sistema a discriminar entre un día normal de trabajo del fin de semana. Toda esta información adicional permitirá mejorar la predicción.

Antes de poder usar el conjunto de datos para entrenar la RNA, estos deben ser preparados. La función de cada una de las RNA del sistema, será clasificar el estado del dispositivo que se está tratando de predecir y controlar (predecir el estado de la lámpara, del calefactor, ventilador y estado de puerta y ventana). Es necesario que se entreguen los casos en que el estado del dispositivo es apagado y en cuales es encendido como vectores de entrada a la RNA.

\section{Preprocesamiento}

Para comenzar, se requiere crear un conjunto de registros para los lúmenes, otro para la temperatura y lo mismo para la humedad, incorporando además, la fecha y hora y el estado de la lámpara, calentador, ventilador, apertura/cierre de puerta y ventana y un valor que indica la presencia o no de personas en la oficina instrumentada. Luego y una vez ya conocidos el rango de lúmenes, temperatura, humedad y ángulos de puerta y ventana que el sistema monitoreará y tratará de predecir, se llenan los espacios en blanco en los registros para obtener un conjunto completo por día (2 por minuto).

El valor que indica la presencia o no de personas en la oficina toma relevancia debido a que si bien los lúmenes o la temperatura están usualmente por debajo del rango considerado como óptimo durante las horas de noche, no es necesario mantener la luminaria o la calefacción encendida en esos horario si no hubieran personas. Lo mismo sucede durante los fines de semana, en donde la presencia de las personas ocurre en un horario distinto al habitual (horarios de aseo). El estado de la puerta y ventana registran también cambios en los patrones en los horarios señalados. La información contenida en los registros se conserva de esta manera para reflejar de forma fiel el uso y horario reales de la oficina. Finalmente el conjunto de datos de entrada (registros), debe ser convertido desde el token a un solo valor. Para esto se conformó un tipo de datos numérico "long" de 64bits, en donde cada dato entrante (lumen, temperatura, fecha, etc.) se alberga en un conjunto subsecuentes de bits de izquierda a derecha. Por ejemplo, los primeros 6 bits más significativos albergarán la temperatura, la cual se podrá expresar en el rango de 0 a $64\left(2^{6}\right)$, puesto a que ese rango permite representar las situaciones de temperatura más habitualmente encontradas.

En la ecuación 1 se realiza la transformación del número binario final al correspondiente en base 10.

$S=\sum_{i=0}^{64} N_{i} * 2^{i}$

En la ecuación, $\mathrm{S}$ representa el número decimal final, el cual se calcula como la sumatoria acumulada del bit $\mathrm{N}$ multiplicado por 2 elevado a la posición del bit. 


\section{Normalización}

La importancia del proceso de normalización radica en que podría acelerar la fase de entrenamiento. Algunos de los métodos de normalización de datos más usados son normalización z-score, min-max, y normalización de escala decimal. Sin embargo para este desarrollo no se utilizó ningún método de normalización, puesto a que tal como se vio, el valor de entrada puede ser transformado desde su representación decimal a binaria y viceversa mediante una simple transformación de base numérica. Un trabajo posterior, una vez probados y validados los rangos, podría verse beneficiado de una transformación lineal de los datos originales mediante el método min-max, puesto que los rangos serian bien conocidos.

\section{RED DE NEURONAS ARTIFICIALES (RNA)}

Con el propósito de obtener la clasificación automática del estado de los aparatos dentro de la oficina instrumentada, se aplicaron redes de neuronas artificiales (feed-forward, conexionado completo, con función de activación sigmoide) (Gurney, 1997) en el conjunto de datos de uso de las luces, calefacción y apertura/cierre de puerta y ventana descrito en las secciones anteriores. Para esto, se utilizó la librería NeuronDotNet, la cual implementa los componentes de RNA necesarios para facilitar el trabajo. Cada vector de entrada contendrá la hora, el día de la semana y un indicador de presencia o no de personas en la oficina. El valor del estado del dispositivo que se está tratando de predecir será utilizado para clasificar los conjuntos de datos.

\section{Estructura}

Se utiliza una RNA por cada dispositivo del cual se pretende obtener su estado de forma automática. Cada RNA utilizada se compone de tres capas (capa de entrada, capa oculta y capa de salida). La capa de entrada tiene 48 neuronas, en donde se codifican los valores del entorno respecto a luminosidad, temperatura, humedad, fecha y presencia o no de personas. Si bien por ejemplo se podría considerar que aspectos como la temperatura o humedad no inciden en la salida que se espera de la red correspondiente a la luz, se optó por mantener esta arquitectura genérica en las redes para permitir un máximo de flexibilidad respecto a los datos de entrada y su posible influencia en otros escenarios respecto a la salida deseada. Así por ejemplo, podemos considerar un escenario diferente como en un invernadero, en donde condiciones de humedad podrían incidir en el encendido/apagado de luminaria de tipo infrarroja. La capa oculta utiliza 4 neuronas. El número de neuronas de la capa oculta no puede ser demasiado pequeño, ya que disminuiría la capacidad de la RNA para aprender patrones, pero si el número de la neurona de la capa oculta es demasiado grande, se corre el riesgo de sobrealimentar el conjunto de datos (Berry, 1997), si bien el número empleado en la capa oculta es pequeño, las pruebas realizadas demuestran que es lo suficiente para obtener una clasificación adecuada. La capa de salida está compuesta por 2 neuronas, lo que permite codificar hasta 4 estados posibles $\left(2^{2}\right)$, de los cuales se utilizarán solo 3 en esta implementación, los que se codificarán como Encender, Apagar y Mantener Estado Actual.

La ecuación 2, muestra como se calcula salida y de la capa de la RNA.

$y=f\left(W_{x}+b\right)$

La función de activación se representa por f(), para lo cual W simboliza la matriz de pesos de las neuronas de la capa, $x$ es el vector de entrada y b es el sesgo del peso de las neuronas.

La función de activación utilizada es la sigmoide, tal como se muestra en la ecuación 3.

$$
f(x)=1 /\left(1-e^{-x}\right)
$$




\section{Entrenamiento}

Para el entrenamiento de la RNA, se utilizo el algoritmo de retro propagación, ejecutando un promedio de 200 iteraciones en cada red. En cada iteración del algoritmo, la forma en la que se calcula la actualización de la matriz w se muestra en la ecuación 4, donde $\gamma$ representa la tasa de aprendizaje y $\delta$ el error de la neurona.

$\Delta w_{i j}=-\gamma \sum_{n} \delta_{j} x_{i}$

Para evaluar la clasificación se utiliza el análisis de sensibilidad (Se) y especificidad (Sp) como medidas estadísticas.

La sensibilidad o tasa recordada, específica la parte de verdaderos positivos (VP) en la clasificación, es decir, en la que se ha detectado correctamente el accionar de un dispositivo, ya sea apagarlo o encenderlo. El (Se) se calcula utilizando la ecuación 5 donde VP (verdaderos positivos) es el número de casos de acción correctamente clasificados y FN (falsos negativos) es el números de errores de clasificación de los casos donde se tiene que actuar y no se ejecuta la acción.

$S e={ }^{V P} /(V P+F N)$

La especificidad es la porción de verdaderos negativos (VN) en todos los casos negativos en el conjunto de datos, es decir, el conjunto de datos en que no se deben accionar los dispositivos. El Sp se determina mediante el cálculo de la ecuación 6, donde VN (verdadero negativo) es el número de clasificaciones correctas para no accionar dispositivos y FP (falso positivo) es el número de errores en el que se clasifican incorrectamente el accionar un dispositivo.

$S P=V N /(V N+F P)$

En la clasificación del estado de acción se encuentran implícitos los estados de encender y apagar, a los cuales se les realiza el mismo análisis utilizando las mismas medidas estadísticas. Cada uno de los valores de Sp necesita ser tan alto como sea posible, ya que la activación errónea de un dispositivo causaría disgusto al usuario, pues posiblemente se abrirá la puerta en un momento no adecuado para hacerlo.

\section{EVALUACION Y RESULTADOS}

Como evaluación de la clasificación de la RNA se determina el accionar de la lámpara y la puerta, el comportamiento de los demás dispositivos es homologable. Para la iluminación se tiene un total de 308.224 registros, de los cuales 4.198 corresponden al encendido, 120.927 corresponden al apagado y 183.099 son para no ejecutar acción. En el caso de la puerta de un total de 7.056 registros, 800 son para la apertura, 2.816 para ejecutar el cierre y 3.440 para mantenerla en el estado en que se encuentra. Las redes se entrenan en un equipo con una PCU de $1.0 \mathrm{Ghz}$, tardando alrededor de 1 hora en promedio, como se ve en la tabla 2.

El alto porcentaje mostrado por Sp en la tabla 3 sobre el $90 \%$ para todas las redes, demuestra que los datos del entorno y los hábitos de las personas están relacionados de alguna forma y las RNA son capaces de clasificarlos exitosamente. Sin embargo debido a la alta cantidad de patrones, aún se presentan patrones que las RNA. Enfocándose en el valor de precisión (Ps) se demuestra que no se puede reconocer un $2.11 \%$ de los patrones del set de entrenamiento y un $0.55 \%$ en el set de entrenamiento de la puerta. 
Tabla 2: Tiempo de entrenamiento

\begin{tabular}{|c|c|c|c|}
\hline Red & Registros & Tiempo & Error \\
\hline lluminación & 308.233 & $60^{\prime} 10 '$ & 0,0147 \\
\hline Ventilación & 37.631 & $58^{\prime \prime}$ & 0,0015 \\
\hline Puerta & 7.055 & $1^{\prime} 19^{\prime \prime}$ & 0,0010 \\
\hline Ventana & 7.039 & $22{ }^{\prime}$ & 0,0012 \\
\hline Calefacción & 3719 & $6^{\prime}$ & 0,0020 \\
\hline
\end{tabular}

Tabla 3: Medidas estadísticas de la clasificación del la RNA para el estado de lámpara y puerta

\begin{tabular}{|c|c|c|c|}
\hline & Sensibilidad (Se) & Especificidad (Sp) & Precisión (Ps) \\
\hline Set entrenamiento Lámpara & 98.64 & 98.55 & 97.89 \\
\hline Set validación Lámpara & 94.92 & 96.69 & 91.80 \\
\hline Set entrenamiento Puerta & 99.39 & 99.42 & 99.45 \\
\hline Set validación Puerta & 90.32 & 99.32 & 98.25 \\
\hline Set entrenamiento Ventilación & 99.65 & 96.77 & 99.79 \\
\hline Set validación Ventilación & 92.65 & 90.02 & 97.54 \\
\hline Set entrenamiento Ventana & 98.68 & 98.65 & 98.65 \\
\hline Set validación Ventana & 91.51 & 93.47 & 97.87 \\
\hline Set entrenamiento & 98.70 & 90.33 & 99.07 \\
\hline Calefacción & 90.91 & 95.48 & 97.51 \\
\hline
\end{tabular}

Una de las funciones de la RNA es tratar de ajustar o generalizar, esto puede explicar la existencia de errores en la clasificación, además los datos en los que se basa el entrenamiento son tomados del entorno real en donde el usuario acciona los dispositivos no siempre de una manera rutinaria, sino ajustando su necesidad de iluminación y calefacción. En el escenario de automatización de la oficina, la clasificación de los datos obtenidos mediante los sensores se realiza durante las 24 horas del día. No es necesario una desactivación del sistema durante la noche debido a que en el conjunto de patrones de entrenamiento existía un número adecuado de patrones nocturnos, en donde no se realizaba ninguna activación de dispositivos (luces, calefacción apagadas, etc.), siendo posible configurar otros escenarios según requerimientos.

Control de lluminación

En el caso de la luminosidad o activación de las lámparas, se determina de forma automática mediante el software, un intervalo en los valores registrados, creando una zona de luminosidad aceptable, como se observa en la figura 5, cuando la lectura de luminosidad esta dentro del rango la RNA determina no activar la lámpara. Si el valor leído sobrepasa la zona aceptable la RNA apaga la iluminación para volver a la zona aceptable. En caso contrario, la RNA activa la luz cuando encuentra que la luminosidad es demasiado baja para el momento.

Comparando con el histograma, se puede validar el encendido de la luz, que solo ocurre en las lecturas bajo los 40 lúmenes. Lo mismo para el apagado de la luz, en donde se comienzan a detectar apagados de la lámpara desde los 150 lúmenes en adelante. Para una correcta clasificación del patrón, se recibe además información sobre la fecha y hora y si la oficina está habitada o no, entre otros. Por ello la luz es encendida también los días sábado y domingo por cortos intervalos de tiempo (horarios de aseo). Un administrador de sistema, puede ajustar los rangos calculados y otras condiciones (horarios, pr ejemplo), de ser requerido para satisfacer 
requerimientos de ahorro energético, seguridad u otros. De lo contrario y por defecto, el sistema utiliza de forma automática la información directamente producida por los hábitos de la persona. Se realizan procesos similares para el caso de control de calefacción y ventilación.

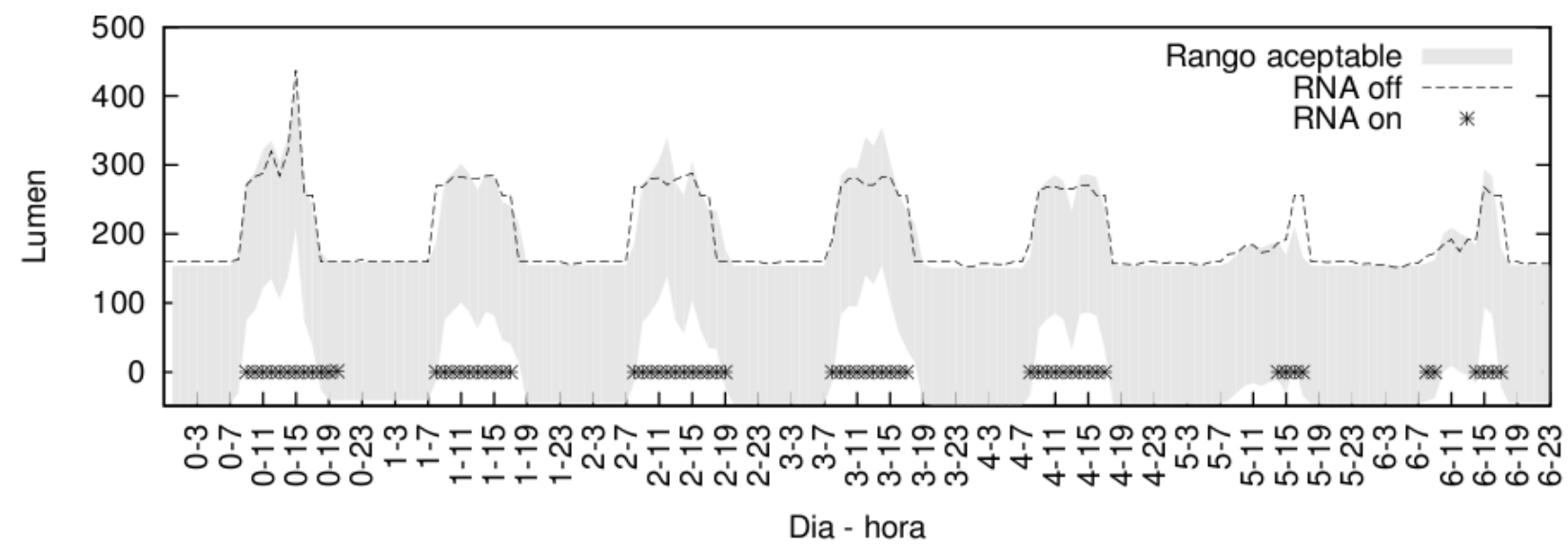

Fig. 5: Luminosidad aceptable semanal y acciones de la RNA

La correcta clasificación y predicción del estado adecuado de estos dispositivos, es importante en términos de confort y ahorro energético, permitiendo combinar los gustos de la persona y los requerimientos energéticos del edificio, logrando un ahorro al mantener la iluminación y calefacción en rangos adecuados sin desperdiciar energía y también apagando o reduciendo el uso de los dispositivos en horarios determinados o en ausencia de personas.

\section{Control de acceso}

Para el caso del control de acceso, figura 6, los valores sobre el umbral determinado se consideran como estado puerta abierta. El comportamiento de la puerta está ligado a los horarios de las personas presentes en el entorno de oficina instrumentada.

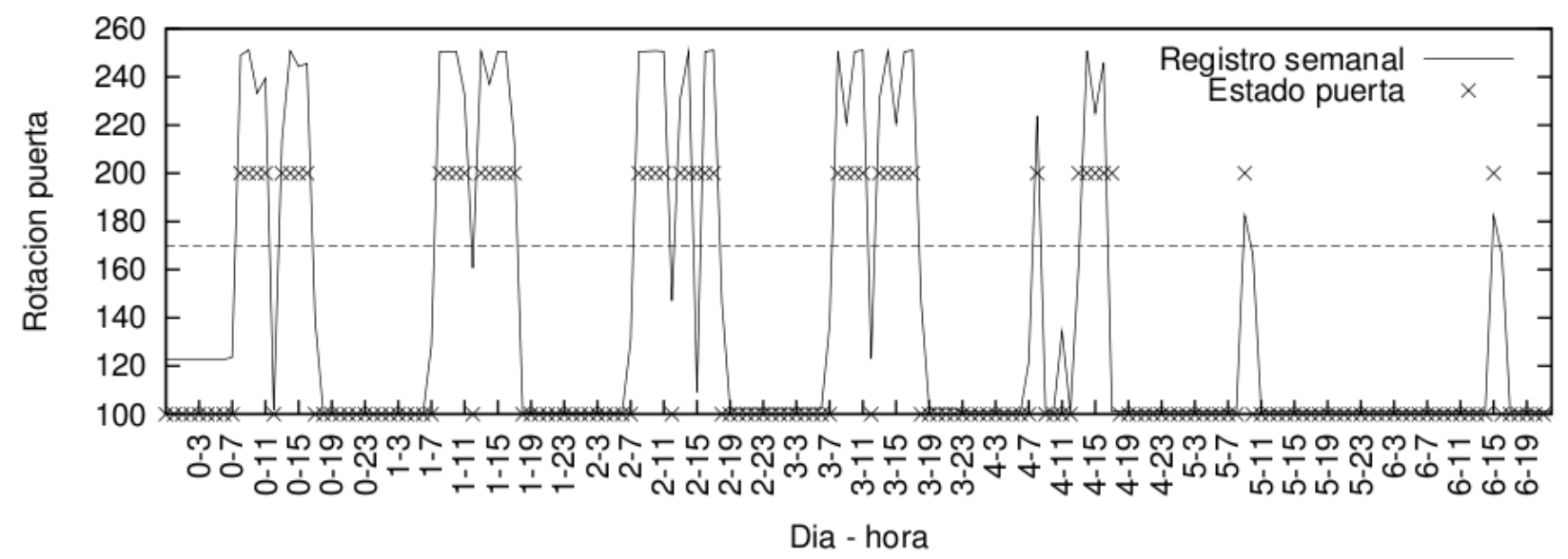

Fig. 6: Registro semanal apertura puerta y acción de la RNA

Comparando con el histograma, se justifican las acciones de aperturas primordialmente en las horas de la mañana y cierres entre 05:00pm y 07:00pm, así como también a las 01:00pm. Para el caso del clasificador RNA para control de la ventana, la clasificación se ve más influenciada por la información relacionada con la humedad y temperatura antes que el horario. Sin embargo el proceso es análogo al presentado para el caso de la puerta. Al igual que en el caso del control de iluminación, un administrador de sistema, puede opcionalmente ajustar las condiciones para cumplir con requerimientos de ahorro energético, seguridad $u$ otros. 
La correcta clasificación y predicción del estado adecuado de los dispositivos puerta y ventana es importante en términos de la seguridad, además del confort, puesto que pueden alertar sobre accesos no autorizados o cerrar el acceso en caso de aperturas accidentales en horarios inadecuados. Se deben entrenar las redes con patrones que se ajusten a los requisitos de seguridad. Otras pruebas realizadas demuestran que este tipo de modificaciones es posible de realizar con la actual estructura de las RNA.

\section{Comportamiento actualizado en el ambiente}

Para detectar los cambios en las estaciones del año y otros cambios en el ambiente, las RNA en el sistema se entrenan constantemente una vez al mes o cuando el usuario lo requiere como un proceso paralelo de baja prioridad por el computador central de la oficina, con el propósito de eliminar patrones antiguos y agregar nuevos. Para el entrenamiento se utilizan registros de los últimos 60 días. Esta frecuencia de entrenamiento es suficiente para mantener el sistema actualizado.

Luego del entrenamiento de las RNA, para cada dispositivo controlado (luces, ventilador, calentador, puerta y ventana), la información es controlada de forma central por el computador de la oficina. Por lo tanto, se requiere de sistemas de respaldo de energía (UPS) o generador para reducir la latencia del sistema dependiendo de lo crítico del contexto.

\section{TRABAJO FUTURO}

El método presentado se aplicará para controlar otros dispositivos en un entorno tipo hogar u oficina tales como: dispositivos multimedia de audio y video, impresoras, scanner, cámaras, proyectores, hornos microondas consolas de juegos, dispensadores de agua, persianas, programas utilizados por el usuario en la computadora (mail, ofimática, navegación internet, redes sociales virtuales, etc.), entre otros. Particularmente dispositivos que poseen un encendido prolongado y no pueden ser simplemente encendidos o apagados. Usualmente se desperdicia energía en tiempos de recarga excesivos en dispositivos móviles o en dispositivos en modo de espera (standby) de forma innecesaria, etc. El confort y la mejora en la eficiencia energética serán los objetivos centrales del control de éste tipo de dispositivos.

En siguientes trabajos, se estudiarán otras estructuras de RNA, más específicas y complejas, con el fin de un mejor Sp y Se. Sin embargo, el método debe ser lo suficientemente simple para poder ser computado de forma paralela por una computadora promedio.

Adicionalmente en trabajos futuros, se puede profundizar en los sistemas de interfaz 3D para casas, haciendo uso de las tecnología de streaming de Icarus 3D para transmitir la interfaz a un dispositivo móvil para el control fuera del lugar por el o los usuarios, la integración de reconocimiento de voz para comandos de voz a distancia, así como la integración virtual en 3D y un sistema de gestos mediante métodos visuales, tal como Microsoft Kinect, con un enfoque que libera totalmente las manos (sin controles remotos o dispositivos similares), e integración más estrecha del entorno virtual 3D y el sistema "plug and play" para la detección y ajuste automático de dispositivos.

La integración con la tecnología streaming para dispositivos móvil, también ofrece la posibilidad de control adicional mediante información posicional de GPS y/o WiFi cuando el usuario este fuera de la oficina o en camino. No basta solo saber que el usuario está en la oficina, sino que también saber que están en camino, podría permitir que el sistema prepare la oficina para su llegada con antelación, logrando una precisión adicional en el uso de energía.

Aunque el método ha presentado resultados muy satisfactorios, otras técnicas de IA serán analizados para comparar su rendimiento y limitaciones. 


\section{CONCLUSIONES}

El trabajo presentado en este documento demuestra que el uso de RNAs para determinar patrones de uso de luz, calefacción, ventilación y uso de puertas y ventanas, es posible mediante la creación de las estructuras de RNA adecuadas.

Mediante las técnicas empleadas, se explota la información registrada de las actividades del usuario, con lo cual se deducen los patrones en un proceso completamente automático.

Una de las principales ventajas de las RNA es su capacidad de generalización, por lo tanto en este escenario, las acciones automáticas deben ser conservadoras y solo actuar cuando exista un nivel de confianza en el desempeño de la RNA.

La arquitectura de RNA del perceptrón multicapa con retro propagación utilizado en este estudio, frente a otras técnicas de I.A o métodos estadísticos, reduce la base de datos de eventos registrados y sus combinatorias de un tamaño de varios megabytes a tan solo unos Kbytes en archivos de entrenamiento compuestos por los pesos de las RNA, lo cual se presenta como una ventaja al momento de utilizar este esquema en computadoras promedio e incluso en dispositivos móviles de características reducidas de hardware.

Un proceso de entrenamiento mensual de la RNA, es suficiente para mantener el sistema actualizado y de esta forma adaptarse a los cambios en el comportamiento de los usuarios causados por las alteraciones de los niveles de luminosidad, temperatura y humedad que se producen por ejemplo en cada estación del año. Aún así, si los usuarios cambian sus patrones rápida y constantemente, el sistema no será capaz de aprender los patrones, lo cual se reflejará como la ausencia de acciones automatizadas en el entorno.

La técnica aquí mostrada, otorga la posibilidad de que un entorno cerrado tipo casa, oficina, bodega, etc, puedan convertirse en sensibles al contexto, debido que al aprender los hábitos de los usuarios en un proceso automático, dota al entorno de la capacidad de ser autónomo y autoconfigurado.

La construcción de un ambiente simulado tridimensional mediante el software lcarus, tanto para el registro de eventos, como en forma de interfaz gráfica de usuario (GUI) para control y monitoreo de los dispositivos, los cual se pueden implementar y configurar de forma rápida, facilita realizar otros estudios respecto a comportamiento humano en entornos cerrados sea posible de llevar a cabo utilizando técnicas de minería de datos sobre los datos registrados, e incluso simularlos sin la presencia física de los usuarios en el entorno. Adicionalmente, los bajos requerimientos de Hardware del entorno virtual, asegura que no se produzcan recargas innecesarias en servidores centrales.

La utilización del Framework para automatización y robótica monoBOTICS, permite que los resultados obtenidos en este trabajo puedan ser utilizados en diversas plataformas y sistemas operativos (Windows, Linux/Unix, Mac OS, Solaris) y con independencia del hardware (diferentes fabricantes y modelos) de captura (sensores) y control de dispositivos (relés, actuadores, etc.).

Según encuestas de satisfacción aplicadas, la computación ubicua se presenta como una técnica capaz de mejorar las relaciones humano-maquina, haciendo que las tecnologías sean más fáciles de manejar de forma integrada en un sistema consciente de su entorno.

\section{REFERENCIAS}

Berry M, Linolf G.; Data Mining Techniques: For Marketing, Sales, and Customer Relationship Management. 2nd Ed. ISBN: 978-0-471-47064-9, (1997). 
Cook D., y otros cinco autores; MavHome: An agent-based smarthome. in Proc. of First IEEE International Conference on Pervasive Computing and Communications (PerCom'03), (2003).

Philips Research - Technology. Disponible (Agosto 2008).

http://www.research.philips.com/technologies/misc/homelab/index.html.

Phidgets: "Set of plug and play building blocks for low cost usb sensing and control from your pc", http://www.phidgets.com.(2010)

monoBOTICS: "Simulation and implementation of robotics and automation solutions" http://monobotics.ic.uach.cl/ (2010)

Icarus: “3D scene engine”, http://pointscapestudios.com (2010)

Kcomt N, y otros cuatro autores: Advances in Soft Computing, Ambient Intelligence and Future Trends-International Symposium on Ambient Intelligencem, Volumen 72, 201-204 (2010).

Morganti G. y otros tres autores: Multi-Agent System Theory for Modelling a Home Automation System, Bio-Inspired Systems: Computational and Ambient Intelligence, Volumen 5517/2009, 585-583 (2009).

Gurney K.; An Introduction to Neural Networks. London: Routledge. ISBN 1-85728-673-1, (1997).

Larson K. y otros cuatro autores; A living laboratory for the design and evaluation of ubiquitous computing technologies. in Proc. of Conference of Human Factors in Computing Systems, April 02-07, Portland, OR, USA, pp.1941-1944.(2005)

Kautz H.,Fox D. y Weld D.; Foundations of assisted cognition systems. University of Washington, Computer Science Department, Technical Report, Tech. Rep., (2003).

Kidd C. y otros nueve autores; The Aware Home: A Living Laboratory for Ubiquitous Computing Research. Proc. of the Second International Workshop on Cooperative Buildings, (1999).

Luihr S., West G. y Venkatesh S.; Recognition of emergent human behaviour in a smart home: A data mining approach. Pervasive Mob. Comput. 3, 2, pp.95-116, (2007).

Machado C. y Mendes J.; Sensors, Actuators and Communicators When Building a Ubiquitous System. in Proc. of IEEE ISIE 2007, in Vigo, Spain, ISBN:1-4244-0755-9 (p1530-1535). (2007)

Hollands R., "The Virtual Reality Homebrewer's Handbook”. John Wiley \& Sons, (1996).

Polikar R; Pattern Recognition, In Wiley Encyclopedia of Biomedical Engineering. Ed. Akay, M., New York, NY: Wiley., (2006).

Rivera-Illingworth F., Callaghan V. y Hagras H.; Automated Discovery of Human Activities inside Pervasive Living Spaces. Proc. of 1st International Symposium on Pervasive Computing and Applications, pp. 77- 82. (2006)

Schmidt A.; Implicit Human-Computer Interaction through Context. In International Symposium on Human Computer Interaction with Mobile Devices and Services (M obileHCl), (1999).

Tapia E, Intille S. y Larson K.; Activity recognition in the home setting using simple and ubiquitous sensors. in Proceedings of PERVASIVE 2004, vol. LNCS 3001, A. Ferscha and F. Mattern, Eds. Berlin Heidelberg: Springer-Verlag, pp. 158-175. (2004)

Weiser M.; The Computer for the Twenty-First Century. In Scientific American, (1991).

Zheng H., Wang H. y Black N. Human Activity Detection in Smart Home Environment with Self Adaptive Neural Networks. in IEEE International Conference Networking, Sensing and Control, ICNSC 2008), ISBN: 978-1-4244-1685-1, pp 1505-1510, (2008) 